\title{
Phase Knife Coronagraph
}

\section{II - Laboratory results}

\author{
L. Abe ${ }^{1,2}$, A. Domiciano de Souza Jr. ${ }^{1,2}$, F. Vakili ${ }^{2}$, and J. Gay ${ }^{1}$ \\ 1 Observatoire de la Côte d'Azur, Département Fresnel, UMR-CNRS 6528, 06460 Saint-Vallier de Thiey, France \\ ${ }^{2}$ UMR-6525 Astrophysique, Université de Nice Sophia-Antipolis, Parc Valrose, 06108 Nice Cedex 02, France
}

Received 28 February 2002 / Accepted 25 October 2002

\begin{abstract}
We report laboratory results from a monochromatic prototype of the Phase Knife Coronagraph which validate our previous theoretical and numerical simulations, prove the physical principle of it and set realistic limitations to the nulling properties of the coronagraph. The optical set-up, phase knife manufacturing technique and different aspects of the instrumental limitations are given. The first results attain easily a 3000 nulling effect obtained both on single and simulated double stars. Optical and mechanical stabilities are discussed and future steps to be carried out for a wide band version of this coronagraph are outlined.
\end{abstract}

Key words. techniques: interferometric- methods: laboratory - stars: binaries: close

\section{Introduction}

Direct detection of exoplanetary systems using both monolithic and multi-aperture synthetic telescopes is amongst one of the most challenging goals of modern observational astronomy and therefore focuses the efforts of major space and/or large ground-based facility agencies such as DARWIN (Leger et al. 1996), TPF (Beichman et al. 1999), NGST (Trauger et al. 1999), Keck-I (Colavita 2001), VLTI (Glindemann et al. 2000). Ultra-high-contrast-imaging at the level of $10^{6}$ to $10^{9}$ and the resolution limit of a 8-10 m class telescope, i.e. 40 to 10 milli-arcsecond (mas) in $K$ to $V$ bands, also paves the way to study the close environment of various classes of astronomical objects from Young Stellar Objects to evolved stars, bringing new insights to verifications of stellar evolution scenarios, for example.

Unlike Lyot's coronagraph, interference nulling coronagraphs (Gay \& Rabbia 1996; Roddier \& Roddier 1997; Rouan et al. 2000) represent the only alternative to attain the resolution limit of present large telescopes like VLT (Monnet 2000), Keck telescopes (Wizinowich et al. 2000) and Gemini (Mountain et al. 1998). This have motivated our efforts to develop the Achromatic Phase-Knife Coronagraph (APKC hereafter, Abe et al. 2001, referred to as Paper I). In this paper we present the first laboratory results from $\mathrm{PKC}$, the monochromatic version of the APKC, as a step along the way to construct a full-fledged high contrast imaging device to use on large ground-based monolithic telescopes equipped with extremely

Send offprint requests to: L. Abe, e-mail: Lyu.Abe@unice.fr dense and high performance Adaptive Optics (A.O. hereafter, Mouillet 2001). In Paper I we described the conditions in which the intrinsic performances of PKC and its achromatic version APKC become similar. Therefore, in order to progress along our plan we have tested a monochromatic laboratory breadboard to validate its physical principle and to obtain realistic numbers on its operational characteristics. Section 2 summarizes the physical concept of the APKC. Section 3 describes phase knife manufacturing and commissioning, and the laboratory optical set-up used to assess its performances. Section 4 details the experimental results obtained on simple and binary simulated stars which settle the nulling performances of the APKC in its present configuration. Section 5 comments on the results and compares them to theory. In the final section we give a critical review of the APKC concept and show how future steps should bring us to a fully operational coronagraph for sky observations. The exact formalism for the residual energy inside the coronagraphic pupil is given in an appendix.

\section{Theoretical background}

\subsection{Summary of the APKC}

The Achromatic Phase Knife Coronagraph concept and its numerically simulated properties were described in our previous paper (Paper I). The APKC is based on an image plane phase mask technique to produce a highly destructive interference pattern into the conjugate pupil plane. It consists of dispersing and $\pi$ phase-shifting the incoming on-axis wave front according to two orthogonal directions in two consecutive image planes (Paper I). The intensity distribution in the conjugate 

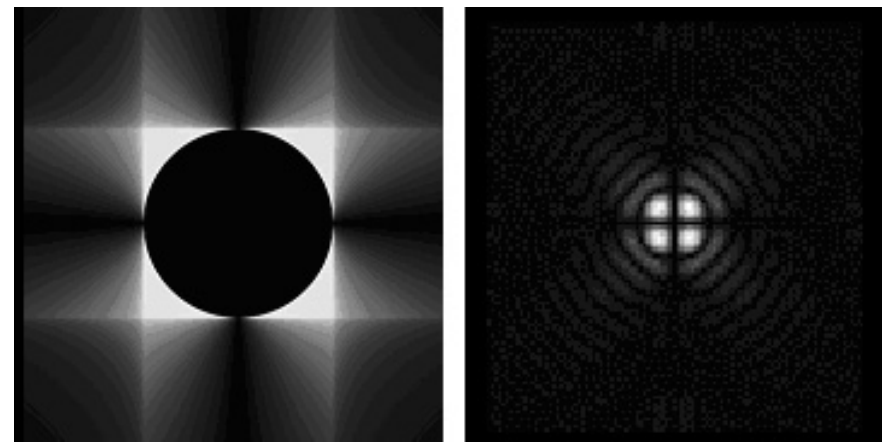

Fig. 1. Theoretical intensity distribution in the pupil plane (left) and in the image plane (right) for a finite field of view.

pupil plane exhibits a quasi-perfect destructive interference exactly matching the pupil shape (Fig. 1, left).

It can be shown (see Appendix A) that in the case of an unobscured telescope, with an infinite image plane phase mask and for a monochromatic wave, the PKC completely rejects the on-axis source energy outside of the conjugate pupil, offering in principle a perfectly nulling coronagraph. Indeed the finite size of the optical components (i.e. a finite field of view) in such a device produces diffraction effects that re-inject energy inside the conjugate pupil. Therefore, the final coronagraphic pattern (Fig. 1, right) exhibits four bright lobes resulting from diffraction residuals. Numerical simulations show that for a $50 \lambda / D$ field, and assuming perfect optics, one can expect a reasonable $10^{6}$ energy rejection for an on-axis source.

In the real world however, imperfect optics, phase retardation properties, atmospheric turbulence and chromatic residues among other degrading effects limit the APKC nulling performances in ways that Sect. 3 will explicitly highlight.

\subsection{Performance criteria}

In any high dynamic imaging process for exo-planet detection, the goal is to increase the local contrast between a central bright star (coronagraphic PSF) and its faint companion. Therefore, the energy rejection does not necessarily represent the best performance criterion. Hereafter we define the more appropriate, to our opinion, criteria to measure the coronagraphic efficiency used throughout this paper:

- extinction: peak-to-peak intensity ratio, defined as:

$$
E=\frac{\max \left(I_{\text {without corona. }}\right)}{\max \left(I_{\text {with corona. }}\right)}
$$

- attenuation factor: the inverse of the extinction $E$

- energy rejection rate: the integrated intensity ratios, either in the image plane:

$$
R_{\text {image }}=\frac{\int_{\text {Full field }} I_{\text {without corona. }}}{\int_{\text {Full field }} I_{\text {with corona. }}}
$$

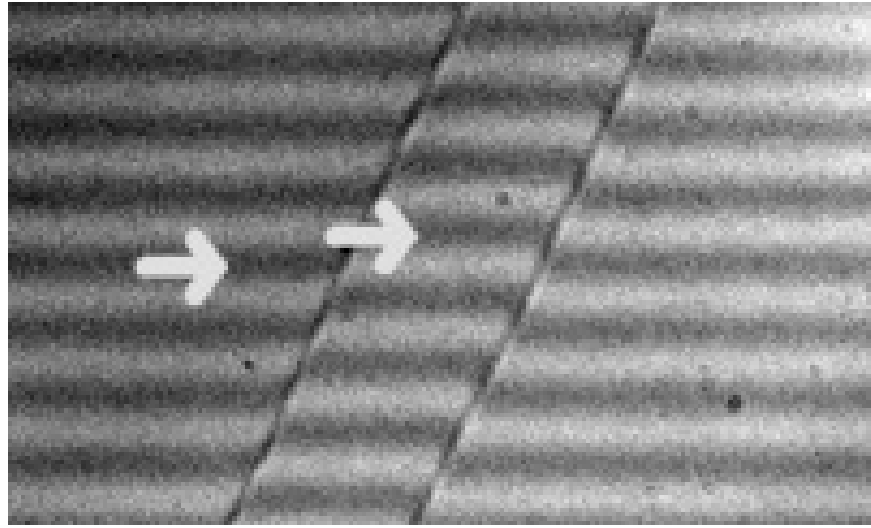

Fig. 2. A phase knife sample seen through an interference microscope. The step between the two fringe patterns from the phase knife zones correspond to a geometrical phase shift which depends on the aluminium deposit thickness (see text for further explanations). The phase-shifts were measured using a $1 \mathrm{~nm}$ bandwidth $\mathrm{H} \alpha$ filter.

or in the pupil plane:

$$
\begin{aligned}
R_{\text {pupil }} & =\frac{T_{\text {Lyot stop }} \int_{\text {Full plane }} I_{\text {with corona. }}}{\int_{\text {Lyot stop }} I_{\text {with corona. }}} \\
& =\frac{\int_{\text {Lyot stop }} I_{\text {without corona. }}}{\int_{\text {Lyot stop }} I_{\text {with corona. }}}
\end{aligned}
$$

where $T_{\text {Lyot stop }}$ is the Lyot stop transmission factor, i.e. the ratio stop-area/pupil-area. The mention with/without corona refers to the focal coronagraphic mask being present or not (all other optical components remaining, including the Lyot stop).

For example, in the case of Fig. 1 (perfect PKC with limited field of view) the rejection rate differs from the extinction ratio by a significant factor of $\gtrsim 4$. More generally, in high dynamic range imaging techniques, the performance is ultimately defined by the achievable local contrast and not by the integrated transmitted energy.

This is the same problem as encountered with the Apodized Square Apertures (Nisenson 2001), the Dark Hole technique (Malbet 1995) and for those instruments that modify the final intensity distribution of a perfectly diffracted Airy pattern.

\section{Laboratory tests and operating conditions}

All tests were carried out with a full, unobscured pupil. The Lyot stop was chosen to be $70 \%$ of the full aperture in order to be less sensitive to optical tuning (stop centering) and residual low order aberrations such as defocus, which appears to be a major limitation to the coronagraph performance.

\subsection{Phase knife manufacturing}

We used common optical glass waveplates on which we deposited aluminium layers. The deposit process was carried out 
at the Observatoire de Nice using a dedicated vacuum tank. The aluminium is heated and evaporated under vacuum with a typical pressure of $2 \times 10^{-6}$ Torr. The glass waveplates were first uniformly coated with an arbitrary thickness. We used vacuumproof adhesive tape to mask a rectangular area on each plate and deposited a second aluminium layer for which the thickness was controlled to match the desired wavelength's phase retardation, for instance HeNe laser $632.8 \mathrm{~nm}$. The final product had a reflective surface with a step-like quarter-wave transition between the two halves of the glass plate. In order to minimize coating differences for a pair of phase knives, we systematically produced two of them at the same time. After a reasonable number of manufacturing trials we obtained a pair of waveplates close to the desired $\pi$-shift of the operating laser wavelength.

\subsection{Surface quality control}

In order to estimate the exact phase shift of the final PKC waveplates, we used an interference microscope. The observed image with such a device corresponds to the superimposition of two slightly displaced images of the same object, on which a fringe pattern is projected. In our case the fringe pattern undergoes a discontinuity at the thickness change location. A typical image of this situation is shown in Fig. 2 where the interference fringes are formed at $\mathrm{H} \alpha$ wavelength $(656.3 \mathrm{~nm}, 1 \mathrm{~nm}$ bandwidth).

Although the two phase knives were built at the same time, they do not produce the same phase-shift on the wave. This is interpreted as being due to a non-uniform coating deposit inside the vacuum tank. For the HeNe laser wavelength $(632.8 \mathrm{~nm})$, we measured the phase-shift difference from the ideal $\pi$ value as $\delta \phi_{1}=(-7 \pm 1) \%$ and $\delta \phi_{2}=(1 \pm 1) \%$ which corresponds respectively to a lack of thickness of $\sim 5.5 \mathrm{~nm}$ and an excess of thickness of $\sim 0.5 \mathrm{~nm}$. All measurements resulted from correlations between the fringe patterns.

The surface quality of the glass waveplates is locally good enough $(<\lambda / 50)$ for our application since we concentrate the light on a very small area of the size of a few Airy discs. Indeed the sharpness and rectilinearity of the step edge directly affect the coronagraphic nulling performance, also limiting the practical size of Airy patterns on the phase knives. The inspection of interferograms showed that edge defects across a few tens of millimeters remain below $10 \mu \mathrm{m}$ at some locations, and the phase transition edge itself extends over less than $2 \mu \mathrm{m}$. Indeed we used such optimum wave-plate zones as much as possible.

\subsection{Optical layout}

Taking into account the physical size of the transition edge and their rectilinearity the optical set-up was dimensioned so as to fit an Airy pattern of about $1 \mathrm{~mm}$ on each phase knife. The large size of the Airy spot prevents the coronagraph performance from being limited by local edge defects, keeping the $\mathrm{PSF} /$ defects size ratio greater than 100 . The source is mimicked by a collimated laser $632.8 \mathrm{~nm}$ beam (see Fig. 3). The collimated beam is relayed by a $\sim 3 \mathrm{~m}$ focal length lens which simulates the telescope entrance pupil and forms an image on the first phase knife. The entrance pupil stop is $5 \mathrm{~mm}$ in diameter and produces an Airy pattern of about $1 \mathrm{~mm}(F / D \approx 640)$, as required. The same magnification is used on the second phase knife which is perpendicular to the first one. The intermediate pupil planes are shown in Fig. 3. A Lyot stop sits at the "coronagraphic pupil" location. A lens focuses the final coronagraphic image on a CCD camera. Digitized frames from this camera are transferred to a host PC for further off-line processing. Each phase knife is mounted on an XYZ Microcontrole plate. The centering of the Airy spot on both phase knives is obtained by push-pulling them in the plane parallel to their surface and perpendicular to the knife edge.

The pin-hole used for the source has a diameter of $10 \mathrm{mi}-$ crons. With the collimating lens, the source is partially resolved by a factor $1 / 16$ th with respect to the Airy radius, roughly corresponding to a 3 mas star observed on a 10 meters telescope in the $K$ band $(2.2 \mu \mathrm{m})$. Note that a partially resolved object can dramatically affect the nulling performance of any coronagraphic device in general and the APKC in particular.

\section{Estimated performances}

\subsection{Coronagraphic pupil intensity distribution}

Figure 4 (bottom) shows the intensity distribution in the final coronagraphic pupil plane obtained by numerical simulation (right) and obtained under present laboratoy conditions (left). We first note the remarkable reproduction of theory by experimental results proving the physical validity of the PKC concept. From this figure one could conclude that the measured rejection should be extremely high but this is due to the CCD intensity dynamic range limitation.

As already noted in Sect. 2.2, and since we could not monitor the pupil image and the PSF simultaneously, we decided to work on the final coronagraphic image rather than measuring rejection rates in the pupil plane. Figure 5 illustrates the nulling effect of the PKC on a binary source. All three images have the same dynamic range with an exposure time of $20 \mathrm{~ms}$. The image on the left shows the normal, i.e. without the PKC, image of a binary source. Placing only one phase knife on the central source (middle) attenuates its intensity already by a factor $\sim 10$ as predicted by theory (corresponding pupil intensity distributions are shown in Fig. 4 (top)). In the right image, the combination of both phase knives almost completely nulls the central bright source. Due to the limited CCD dynamic range ( 8 bits) for a single exposure the signal to noise ratio is not high enough to correctly assess the coronagraphic PSF. Note also that the binary component remains unaffected by the coronagraph at this location. According to Paper I, for an off-axis companion falling exactly on the edge of one of the two PKC knife edges, the effect would be identical to the one of the bright source in the middle image of Fig. 5.

\subsection{The coronagraphic PSFs}

In order to estimate the attenuation factor of the PKC, we must measure the fluxes of the source with and without the 


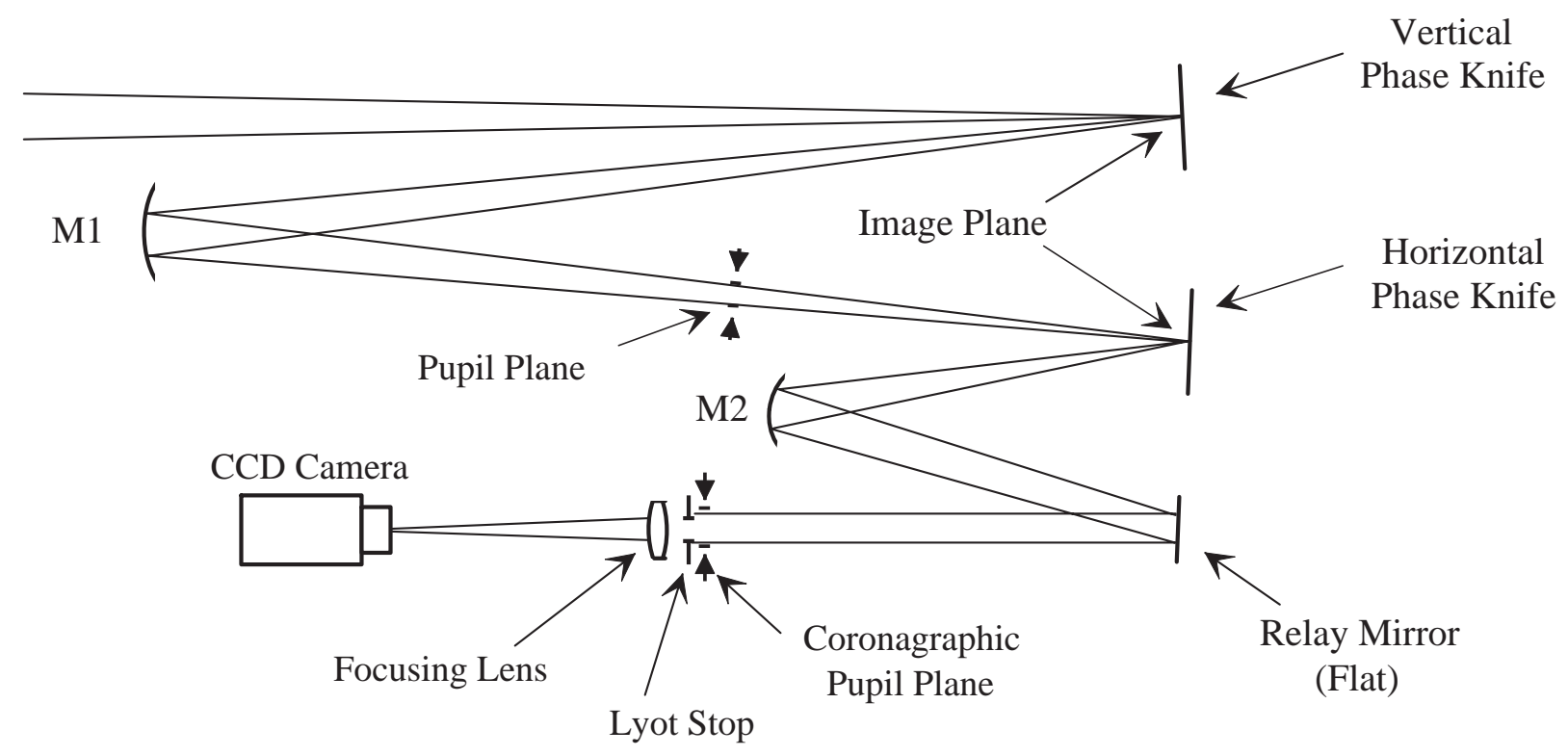

Fig. 3. PKC optical layout. The two phase knives (horizontal and vertical) are equivalent to flat mirrors. M1 and M2 are spherical, forming the second focus and the final coronagraphic pupil image respectively.

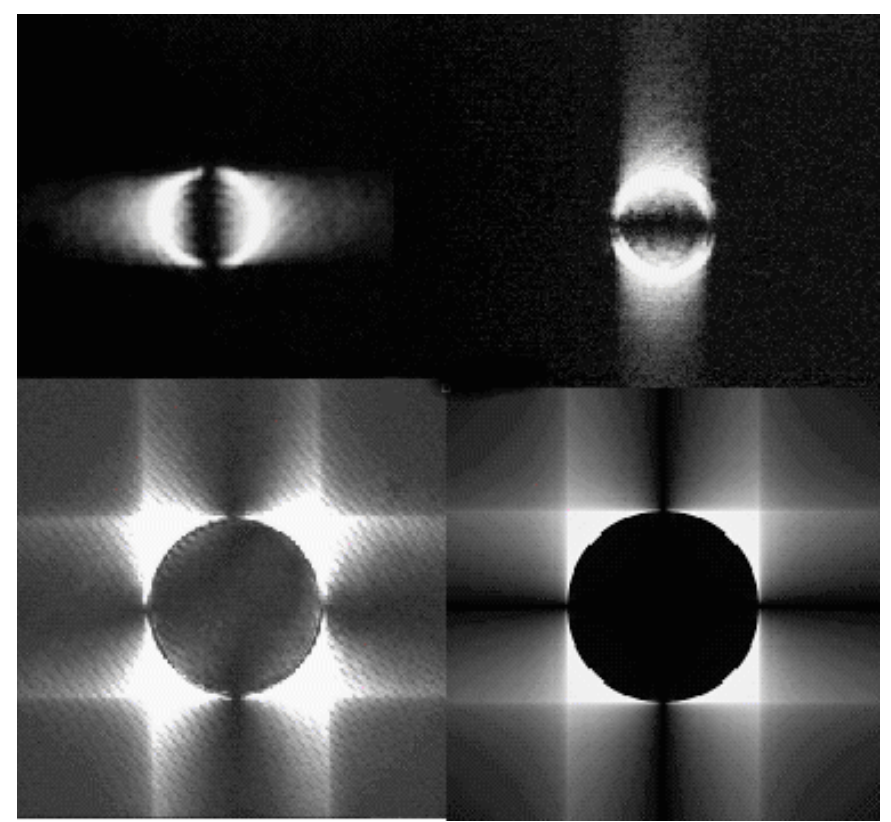

Fig. 4. Comparison of actual laboratory and theoretical intensity distribution in successive pupil planes of the PKC coronagraph (not same scales): (top-left) pupil intensity after the vertical phase-knife alone, (top-right) pupil intensity after the horizontal phase-knife alone, (bottom-left) pupil intensity after both phase-knives being applied and (bottom-right) theoretical pupil intensity distribution. Note that for the actual pupil intensities the CCD exposure has been saturated to show the depth of the null inside the coronagraphic pupil. Fringes on the experimental data are due to multiple reflections on the CCD window.

coronagraph. To avoid CCD saturation for the off-axis source, we used a neutral density filter $(D \approx 3)$ before the laser beam. To overcome the lack of dynamic range of the CCD camera when observing the coronagraphic image, we removed this density filter.

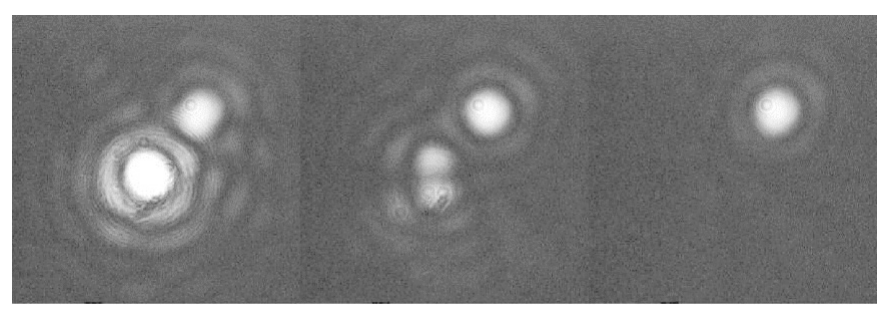

Fig. 5. Application of the two-step PKC phase-knives to a laboratory binary source: left) the binary star star without coronagraph, middle) effect of the first horizontal phase-knife on the central bright star and right) the on-axis star being nulled after the second vertical phaseknife is applied. Images are shown with a non-linear intensity scale to enable direct comparison with the background noise.

When removing the density filter, the coronagraphic PSF was still bright enough to match the CCD dynamic range for a short exposure of $20 \mathrm{~ms}$. Consequently, it was not necessary to carry out time-consuming long exposures to attain the desired SNR. We could also monitor the effects of the tip-tilt jitter on the coronagraphic PSF while recording a series of short exposures. Indeed, the temperature inside the laboratory was relatively stable and produced only low order phase aberrations, using Zernike polynomials terminology, resulting in fast variations of both shape and intensity of the coronagraphic PSF.

\subsection{Image selection and final maximum nulling effect}

Using a fast read-out detector and short exposures allows the selection of the best images in the whole sequence, i.e. images where the coronagraphic PSF flux of the on-axis source is lowest. Figure 6 (top) shows the intensity fluctuations of the brightest lobes of the coronagraphic PSF. As mentioned before, tip-tilt is mainly responsible for such variations. We could derive the tip-tilt errors from the recorded sequence of a reference Airy pattern without the coronagraph. The pixel 


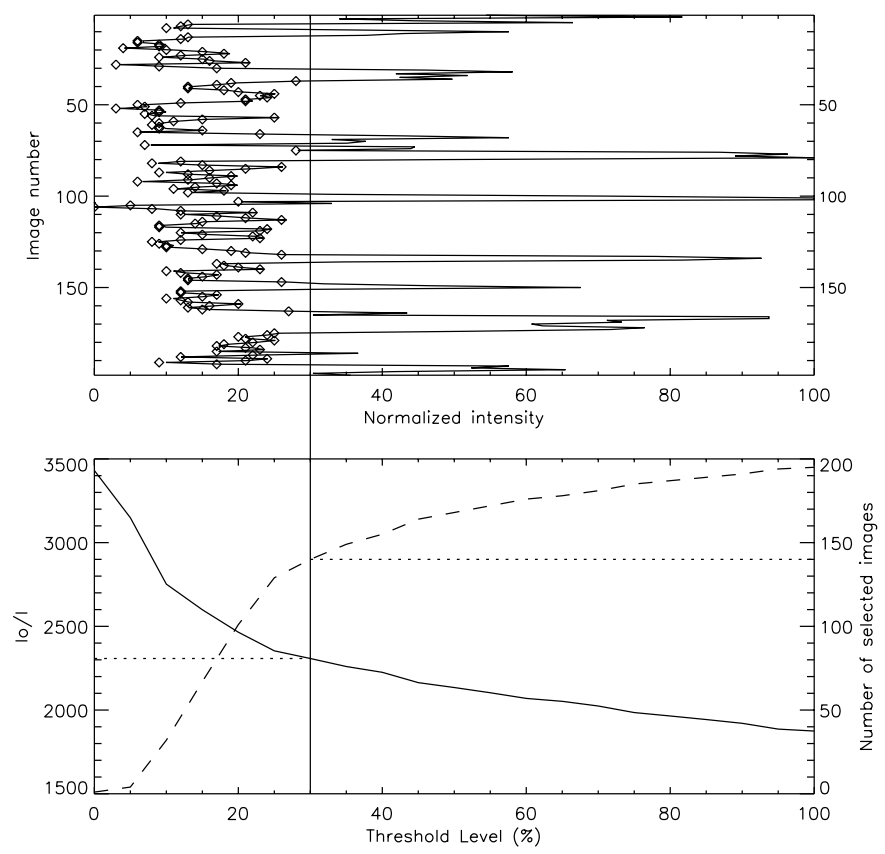

Fig. 6. (top) Coronagraphic PSF intensity maxima recorded during a continuous series of 199 exposures (pixel intensity digitized over 8 bits). (bottom) Image selection procedure applied to the exposure set according to a flux threshold defined as a percentage of the maximum intensity variation (full dynamic range of top). The solid curve is the peak-to-peak intensity ratio (the extinction, noted $I_{0} / I$ ) between the off-axis reference Airy pattern and the integrated coronagraphic PSFs selected by applying the threshold. The dashed curve gives the number of selected images after thresholding. In the given example, the 140 selected images are marked with diamonds on the top figure for a threshold level of $30 \%$, resulting in a long exposure extinction of $\sim 2300$.

sampling of the images enabled us to precisely compute the photometric centroid of the reference PSF, which turned out to be about $2.4 \%$ rms of the Airy pattern lobe diameter. This corresponds to an amplitude error of $\lambda / 100$ reported on the wavefront. Lower tip-tilt jitter would require stabilized pressure and temperature, and dust free atmosphere (see comments on that subject in Ollivier 2001).

We applied various selection criteria to the image sequence (Fig. 6, bottom) to determine how the overall extinction is affected by "bad" images (i.e. with low extinction). The average extinction ratio for the long exposure for these 199 frames is 1870 . Selecting half the images in the sequence results in a $\sim 40 \%$ gain for the extinction. Results show that we can increase the extinction up to a factor 2 in the best case. However, the optimum selection criterion results from a good compromise between rejection rate and the signal to noise ratio for a possible off-axis object.

\section{Discussion of laboratory results}

In the scope of determining the coronagraph's sensitivity to both phase-shift defects and tip-tilt jitter, we tried to reproduce the experimental conditions with a numerical simulation.

In a first step, we focused on pure optical and turbulence aberrations considering perfect phase knives: static optical aberrations derived from ray-tracing analysis, and numerical simulations show no significant effect on the final coronagraphic extinction. We conclude that the main degrading aberration is a systematic de-centering (constant shift) of the central source.

Then for turbulence aberrations we introduced tip-tilt jitter derived from the off axis reference PSF measurements: we used a Gaussian statistic with a $1 \%$ rms amplitude in $\lambda / D$ units (see Sect. 4.3) and a mean position exactly centered the coronagraph's axis. The phase knives were first considered perfectly $\pi$-shifting. The same image selection procedure as for the experimental data was used, as shown in Fig. 7 (top). This graph indicates that only $\sim 10 \%$ of the images have a very good extinction, greater than $5 \times 10^{5}$, with a maximum at $\sim 10^{6}$ as predicted by the perfect static model.

Now, when introducing the measured phase-shift defects on both knives, and using the same image selection (Fig. 7, bottom), the expected maximal extinction is $\sim 3370$, with a long exposure extinction of $\sim 3070$. This significant loss of performance is rather close to our experimental results. We also notice that the proportion of images with a good extinction is much higher and always very close to the maximum extinction which means that the extinction variance (in percentage) is much lower than in the perfect case.

We conclude that the dominant degradation comes from imperfect phase-shift on the phase knives rather than the tip-tilt jitter.

Results of Fig. 7 (top) are better than the experimental ones (Fig. 6), but the assumption we made of a perfectly centered spot on the coronagraph axis is a too restrictive hypothesis, since we could not verify it during the experiments. By introducing a centering error (in this case for example $8 \%$ of $\lambda / D$ in the vertical direction), we can almost reproduce the same statistics (Fig. 8) and intensity distribution (Fig. 9). Such an error on the centering appears to be rather large compared to the manual tuning precision of each individual optical element (the two phase knives). Nevertheless, there are other tuning error sources (e.g. turbulence instabilities, visual estimations of the residual flux, separate manual tuning for each phase knife) that we could not monitor in real-time. Therefore we point out that for an optimized PKC one requires an extremely accurate and robust tuning procedure.

\section{Conclusion}

The results of the present prototype of the APKC agree surprisingly well with theory and can easily attain an extinction ratio of 3000. In our laboratory experiments we used phase knives which were not perfect $\pi$ phase-retarders. We showed that even in this case the nulling performance was dominated by phase-shift errors rather than centering errors. The latter includes tip-tilt jitter and systematic de-centering, originating respectively from turbulence fluctuations in the laboratory and manual positioning of the optics. The relative size of the Airy pattern $(1 \mathrm{~mm})$ compared to the phase transition zone of less than $2 \mu \mathrm{m}$ (described in Sect. 3) does not seem to produce any significant effect. 

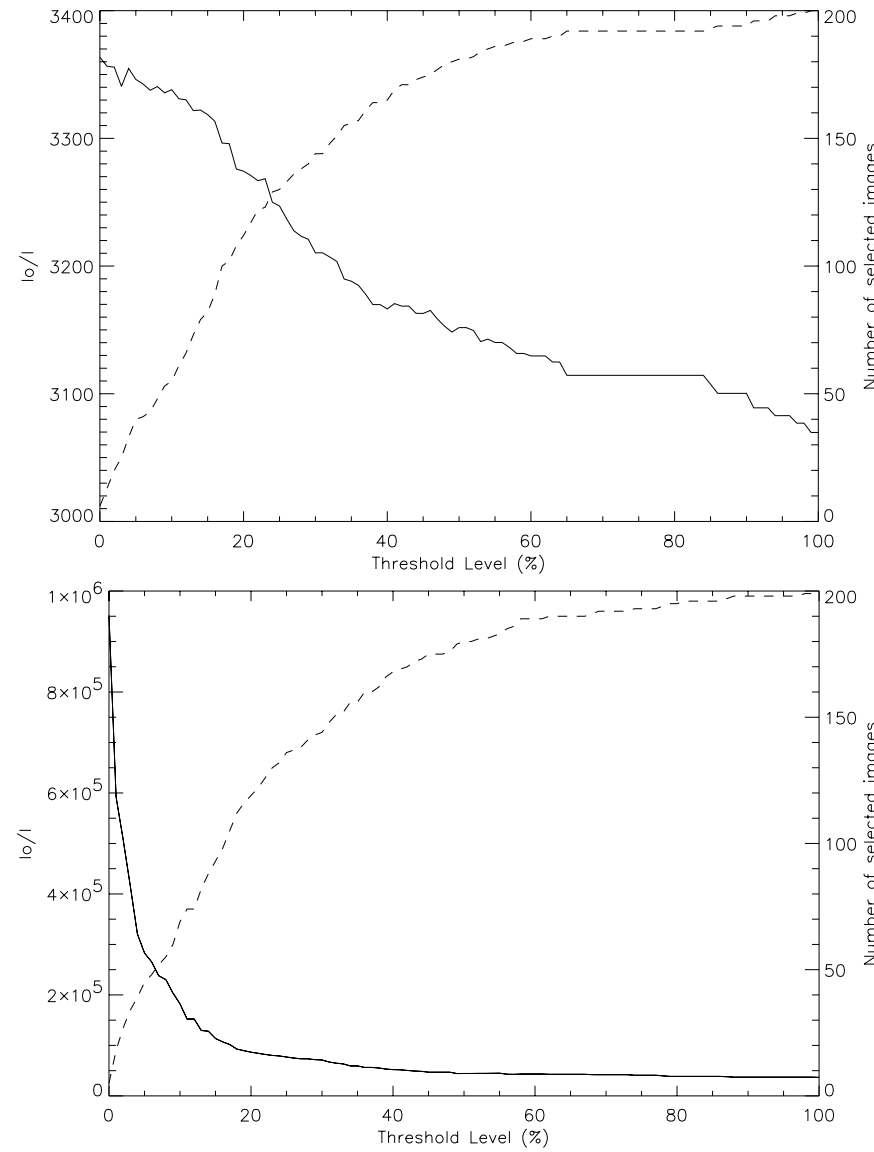

Fig. 7. (top) Image selection process of 200 numerically computed images where the experimental conditions were introduced (tip-tilt jitter and phase knives defects). (bottom) Same as top with tip-tilt jitter only. The Airy spot is perfectly centered on the coronagraph's axis with a gaussian tip-tilt jitter of $1 \%$ rms $(\lambda / D$ units $)$.

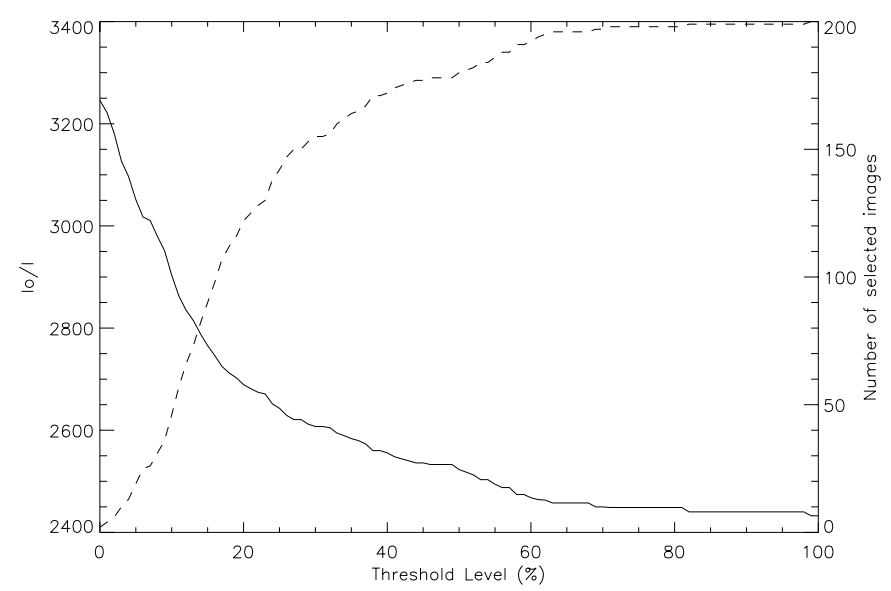

Fig. 8. Same curve as Fig. 7 but where a centering error of $8 \%$ of the Airy spot on the coronagraph axis has been introduced.

This study also shows that the optimal phase knife manufacturing quality depends on the observing strategy and $\mathrm{AO} /$ detector capability: different tip-tilt jitter and phase-shift defect combinations can result in an identical integrated extinction (i.e. summing all exposures). For example, in our case, a $1.5 \%$ rms tip-tilt jitter $(\lambda / D$ units $)$ associated with perfectly
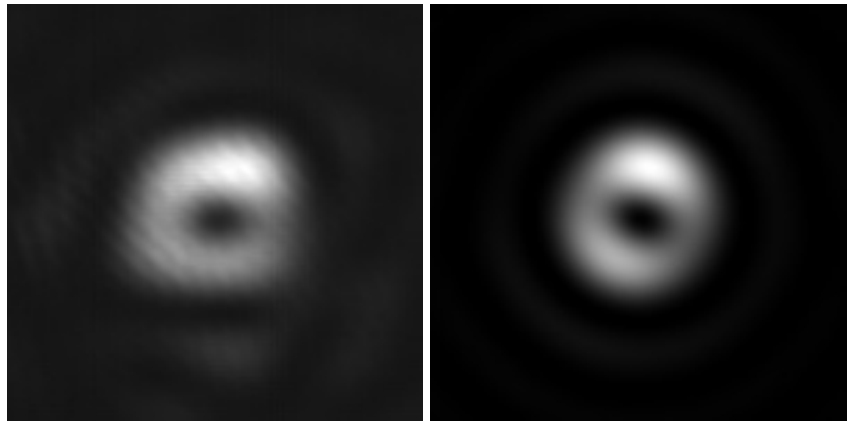

Fig. 9. Long exposure images of (left) the experimental data and (right) simulated data, corresponding to the graph of Fig. 8. Notice that the bright bump location on top of the ring on both images almost exactly match. It mainly originates from the important phase-shift defect $(\sim 7 \%)$ of the first knife. Images are shown in linear intensity map.

phase-shifting knives roughly gives the same integrated extinction $\left(5 \times 10^{4}\right)$ as a $0.5 \%$ jitter and a $1.0 \%$ phase knife defect. On the contrary, if an image selection strategy is chosen using a very fast and sensitive detector (e.g. a photon-counting camera), then top quality manufacturing will be mandatory to allow the optimal performance of the coronagraph. Note that in this case if the flux is very low, one needs to perform quite long exposures, losing the possibility to access a statistical analysis of the data in order to select the best images. Therefore the importance of sensitive and fast read-out detectors for coronagraphic instruments, such as fast photon counting cameras or new Low Light Level CCDs (Mackay 2001; Jerram 2001) becomes more clear.

These considerations should be taken into account for a future APKC in order to significantly improve its performances relative to the present operating prototype and to approach the theoretical nulling effect of $10^{6}$, specially for a wide-band version. The phase knife manufacturing for the APKC concept as described in Paper I differs from this achromatic version by the prismatic shape to be given to the knives. The end-to-end rectilinearity of the phase transition should be kept less than a few percent of $\lambda / \mathrm{D}$ as well as local edge defects. A more complete study should be carried out in order to estimate the sensitivity to these types of defects.

The tip-tilt should be monitored independently of the main AO loop using the rejected light outside the coronagraphic pupil. It should be noted that fine tip-tilt compensation in APKC can be achieved by equipping the two phase knives (see Fig. 2 of Paper I) independently with a single fast linear motion actuator. For a $1 \mathrm{~mm}$ diameter Airy spot, such an actuator should have a typical dynamic range of a few tens of microns with a linear resolution of one micron corresponding to a tip-tilt fine compensation better than $\lambda / 50$. On the other hand the combination of two successive reflection phase-knives in APKC and the exact chromatic phase-retardation compensation by means of diffraction gratings can be applied for IR observations. This characteristic combined with the dispersing/dedispersing technique to obtain an achromatic PKC represent a straightforward way to transform the APKC to a spectrometric coronagraph. This is particularly adapted to spectrally characterize an exoplanet once it has been detected in the wide band 
configuration of APKC. At the present time we have begun the commissioning of a hybrid version of the APKC and the 4QC (Rouan et al. 2000). The coronagraph will be tested in sky observation runs using a photon-counting camera and image selection as described above. The exact coronagraph configuration and the results will be discussed in a future paper.

We conclude that although a space-borne telescope appears as the natural site to implement an APKC, a ground-based instrument allows direct imaging of $\Delta m \approx 6$ with realistic turbulence and residual tip-tilt errors of $\$ 10 \%$ (Paper I, Rouan et al. 2000). Benefiting from our laboratory results, the APKC concept clearly constitutes a potential candidate for future groundbased adaptive optics plus coronagraphic instruments for exoplanet hunting similar to the VLT-Planet Finder foreseen in the near future on an $8 \mathrm{~m}$ telescope.

\section{Appendix A: Mathematical formalism}

A first mathematical analysis was done in Paper I, but did not completely describe the whole nulling effect. Its final coronagraphic principle is formally identical to the 4QC. One of us (J. Gay) developed the complete mathematical formalism of this type of coronagraphic device for a circular pupil of radius $R$ without a central obscuration, which is given in the following. It is demonstrated that in the case of an infinite field image plane, the extinction can be total. Although it is not physically realistic, it means that the extinction can be arbitrarily good depending on the field of a "sectorized" phase-mask coronagraph.

In the pupil plane, we express the spatial frequency coordinates as:

$\eta=\rho \cos (\vartheta)$

and

$\xi=\rho \sin (\vartheta)$

where $\rho$ and $\vartheta$ are the polar coordinates in the reference system centered on the optical axis.

As already mentioned in Paper I, the amplitude distribution is the result of the convolution between the entrance pupil function and the Fourier transform of the PKC function $\operatorname{pkc}(x, y)=$ $\operatorname{sign}(x) \operatorname{sign}(y)$ in the infinite field case which can be written as,

$P_{\mathrm{v}}(\eta, \xi)=P_{\mathrm{v}}\left(-\frac{1}{\pi^{2} \eta \xi}\right)$

and rewritten in polar coordinates,

$P_{\mathrm{v}}(\rho, \vartheta)=P_{\mathrm{v}}\left(-\frac{2}{\pi^{2}} \frac{1}{\rho^{2} \sin (2 \vartheta)}\right)$.

This distribution is usually referred as Cauchy's principal value $\left(P_{\mathrm{v}}\right)$.

We express the integration process in the sense of the principal value by excluding from the integration domain the symmetric areas surrounding the discontinuities as shown in Fig. A.1. The radius $\varepsilon$ and the angle $\alpha$ respectively prevent divergence at the center point and along the $\eta$ and $\xi$ axis. These parameters will tend to zero once the result of the convolution product is established.

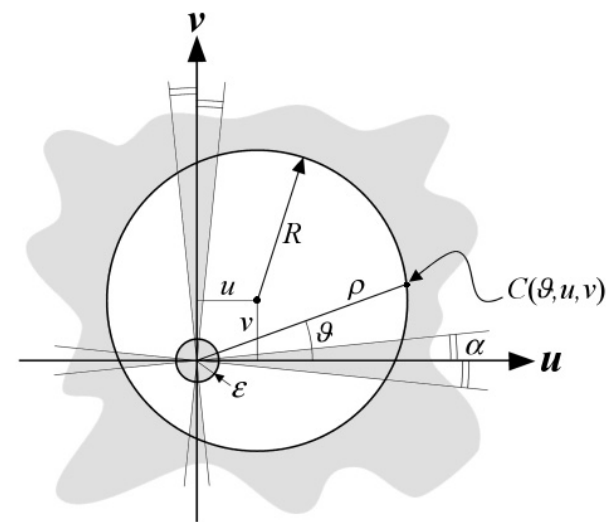

Fig. A.1. Integration domain is delimited by the pupil circle. Gray areas are excluded from it.

Using Eq. (A.2), the convolution product $A=\Pi \otimes P_{\mathrm{v}}$ is written

$A(u, v)=\int_{\eta} \int_{\xi} P_{\mathrm{v}}(\eta, \xi) \Pi(u-\eta, v-\xi) \mathrm{d} \eta \mathrm{d} \xi$

with $\Pi$ being the pupil function which takes the value 1 within the pupil limit, and zero outside. By changing $\eta$ and $\xi$ to polar coordinates, Eq. (A.3) becomes,

$A(u, v)=-\frac{2}{\pi^{2}} \int_{\vartheta} \frac{1}{\sin (2 \vartheta)} \int_{\rho} \Pi(u-\rho \cos (\vartheta), v-\rho \sin (\vartheta)) \frac{\mathrm{d} \rho}{\rho} \mathrm{d} \vartheta$.

Since we are only interested in the intensity distribution inside the pupil area, we only consider the points verifying $u^{2}+v^{2} \leq$ $R^{2}$. Independently, for each of these points, the upper limit of the integral over $\rho$ can be expressed as $C(\vartheta, u, v)$ which describes the circumference of the pupil. White areas in Fig. A.1 show the final integration domain. Therefore,

$$
\begin{aligned}
A(u, v) & =-\frac{2}{\pi^{2}} \int_{\vartheta} \frac{1}{\sin (2 \vartheta)}\left(\int_{\rho=\varepsilon}^{C(\vartheta, u, v)} \frac{\mathrm{d} \rho}{\rho}\right) \mathrm{d} \vartheta \\
& =-\frac{2}{\pi^{2}} \int_{\vartheta} \frac{1}{\sin (2 \vartheta)}[\ln (C(\vartheta, u, v)-\ln (\varepsilon)] \mathrm{d} \vartheta .
\end{aligned}
$$

Then we perform the integration over $\vartheta$ from $\alpha$ to $\pi / 2-\alpha$ by gathering the contributions over $\rho$ between $\varepsilon$ and $C(\vartheta+$ $k \pi / 2, u, v$ ) (with $k=0,1,2,3$ ), taking into account for each $k$ the sign of $\sin (2 \vartheta+2 k \pi / 2)$, which finally leads to,

$$
\begin{aligned}
A(u, v)= & -\frac{2}{\pi^{2}} \int_{\vartheta=\alpha}^{\frac{\pi}{2}-\alpha} \frac{1}{\sin (2 \vartheta)}\{[\ln (C(\vartheta, u, v)-\ln (\varepsilon)] \\
& -[\ln (C(\vartheta+\pi / 2), u, v)-\ln (\varepsilon)] \\
& +[\ln (C(\vartheta+2 \pi / 2), u, v)-\ln (\varepsilon)] \\
& -[\ln (C(\vartheta+3 \pi / 2), u, v)-\ln (\varepsilon)]\} \mathrm{d} \vartheta .
\end{aligned}
$$

Contributions from $\varepsilon$ vanish leaving only the contributions from $C$, therefore

$$
A(u, v)=-\frac{2}{\pi^{2}} \int_{\vartheta=\alpha}^{\frac{\pi}{2}-\alpha} \frac{1}{\sin (2 \vartheta)} W(\vartheta, u, v) \mathrm{d} \vartheta
$$




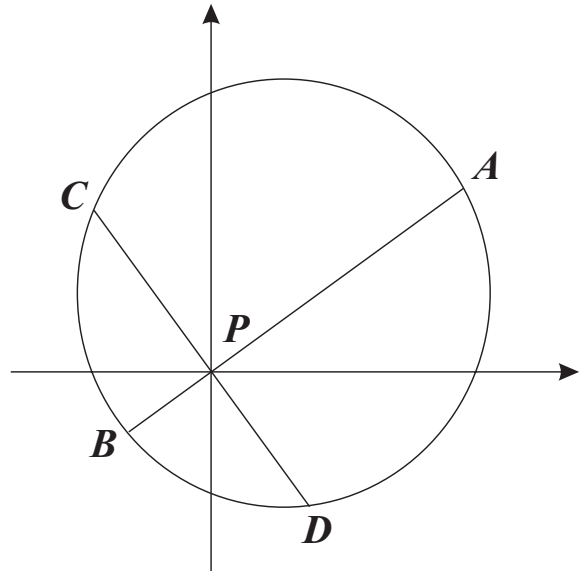

Fig. A.2. Power of point $P$ applied to the the four integration areas described in the text.

with,

$W(\vartheta, u, v)=\ln \left\{\frac{C(\vartheta, u, v) C(\vartheta+\pi, u, v)}{C(\vartheta+\pi / 2, u, v) C(\vartheta+3 \pi / 2, u, v)}\right\}$

and referring to Fig. A.2,

$W(\vartheta, u, v)=\frac{\overline{P A} \cdot \overline{P B}}{\overline{P C} \cdot \overline{P D}}$

One can recognize in $\overline{P A} \cdot \overline{P B}$ or $\overline{P C} \cdot \overline{P D}$ the power of point $P$ relative to the circle which limits the pupil area (Fig. A.2): this is a constant that only depends on the position of point $P$ (see Appendix B). It results in the logarithm argument being always equal to unity, thus cancelling $A$ at any point $(u, v)$ and for any values of $\varepsilon$ and $\alpha$, even tending to zero. We conclude that the energy within the coronagraphic pupil for an infinite field is completely nulled.

\section{Appendix B: Power of a point relative to a circle}

By a point $P$ of the plan which contains a circle of center $O$ and radius $R$, we draw a line intersecting the circle at points $A$ and $B$. The perpendicular to $A B$ passing through $O$ intersects the segment $A B$ in its middle point $M$ (Fig. B.1).
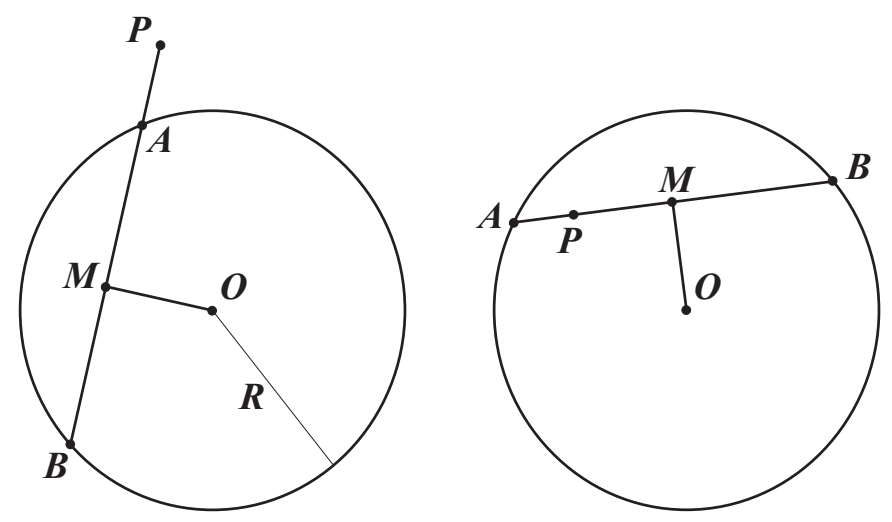

Fig. B.1. The power of point $P$ relative to the circle $\{O, R\}$ can be defined for any point of the plan.
The product $\overline{P A} \cdot \overline{P B}$ can be decomposed as,

$\overline{P A} \cdot \overline{P B}=\overline{P M}^{2}+\overline{P M} \cdot(\overline{M A}+\overline{M B})+\overline{M A} \cdot \overline{M B}$

but,

$\overline{M A}+\overline{M B}=0$

and

$\overline{M A} \cdot \overline{M B}=-\overline{M B}^{2}=-\left(\overline{O B}^{2}-\overline{O M}^{2}\right)$

$\overline{P M}^{2}=\overline{P O}^{2}-\overline{O M}^{2}$.

Consequently,

$\overline{P A} \cdot \overline{P B}=\overline{P O}^{2}-\overline{O B}^{2}=L^{2}-R^{2}$

where $L$ is the distance of point $P$ to $O$. This result shows that this product is independent of the initial line intersecting the circle, and is called the power of point $P$ relative to the circle $\{O, R\}$.

Acknowledgements. L. Abe is grateful to R. Krawczyk and Alcatel-Space Industries/Cannes for supporting his $\mathrm{PhD}$ fellowship. A.Domiciano de Souza Jr. acknowledges CAPES - Brazil (contract BEX 1661/98-1) for financial support. APKC development is supported by the ASHRA-CNRS program in France. The authors would like to thank the following people for their assistance in the APKC laboratory prototype realization: P. Assus, Y. Bresson, F. Desenfant, A. Glintzlin, J.-L. Schneider, A. Spang and N. Thureau. This paper benefitted from critical discussions with C. Aime, L. Arnold, D. Bonneau, A. Labeyrie, D. Mourard, Y. Rabbia, R. Soummer, E. Thomas and D. Vernet.

\section{References}

Abe, L., Vakili, F., \& Boccaletti, A. 2001, A\&A, 374, 1161

Beichman, A., Woolf, N. J., \& Lindensmith, C. A. (ed.) 1999, Terrestrial Planet Finder (Pasadena: JPL Publication 99-3)

Colavita, M. 2001, AAS, 198, 5102C

Gay, J., \& Rabbia, Y. 1996, CR. Acad. Sci. Paris, t. 332, Série IIb, $665-271$

Glindemann, A., et al. 2000, SPIE Proc., 4006, 2

Jerram, P., Pool, P., Bell, R., et al. 2001, Marconi Applied Technologies, Chelmsford, Essex, UK

Leger, A., Mariotti, J. M., Mennesson, B., et al. 1996, Ap\&SS, 241, 135

Malbet, F., Yu, J. W., \& Shao, M. 1995, PASP, 107, 386-398

Mackay, C. D., \& Tubbs, R. N. 2001, Sub-Electron Read Noise at MHz Pixel Rates, SPIE Conf. Proc., 4306, 289

Monnet, G. J. 2000, SPIE Proc., 4008, 8

Mouillet, D., Beuzit, J.-L., Chauvin, G., et al. 2001, in Workshop on Scientific Drivers for ESO Future VLT/VLTI Instrumentation, (Garching: ESO).

Mountain, C. M., Gillett, F. C., \& Oschmann, J. M. 1998, SPIE Proc., 3352, 2

Nisenson, P., \& Papaliolios, C. 2001, AJ, 548, L201

Ollivier, M., Mariotti, J.-M., Léger, A., et al. 2001, A\&A, 370, 1128

Roddier, F., \& Roddier, C. 1997, PASP, 109, 815

Rouan, D., Riaud, P., Boccaletti, A., Clénet, Y., \& Labeyrie, A. 2000, PASP, 122, 1479

Trauger, J., Backman, D., Beichman, C., et al. 1999, Bull. Am. Astron. Soc., 31, 834

Wizinowich, P., et al. 2000, PASP, 112, 315 